\title{
Frequency versus time domain immunity testing of Smart Grid components
}

\author{
F. Gronwald \\ Germany \\ Correspondence to: F. Gronwald (gronwald@tuhh.de) \\ Received: 24 January 2014 - Accepted: 28 February 2014 - Published: 10 November 2014
}

Institute of Electromagnetic Theory, Hamburg University of Technology, Harburger Schloss Str. 20, 21079 Hamburg,

\begin{abstract}
Smart Grid components often are subject to considerable conducted current disturbances in the frequency range $2-150 \mathrm{kHz}$ and, as a consequence, it is necessary to provide reliable immunity test methods. The relevant basic standard IEC 61000-4-19 that is currently under discussion focusses on frequency domain test methods. It is remarked in this contribution that in the context of frequency domain testing the chosen frequency spacing is related to the resonance response of the system under test which, in turn, is characterized in terms of resonance frequencies and quality factors. These notions apply well to physical system but it is pointed out by the example of an actual smart meter immunity test that smart grid components may exhibit susceptibilities that do not necessarily follow a resonance pattern and, additionally, can be narrowband. As a consequence it is suggested to supplement the present frequency domain test methods by time domain tests which utilize damped sinusoidal excitations with corresponding spectra that properly cover the frequency range $2-150 \mathrm{kHz}$, as exemplified by the military standard MIL-STD-461.
\end{abstract}

\section{Introduction}

In view of Electromagnetic Compatibility (EMC) of smart electrical energy networks, the so-called "smart grids", there are strong concerns about the frequency range $2-150 \mathrm{kHz}$. It has been observed that smart grid components, such as smart energy meters, can be subject to conducted disturbances that leads to malfunction, even though the components comply to the mandatory EMC tests (Kirchhof, 2010; CENELEC SC 205A, 2010, 2013). This situation is mainly due to insufficient EMC standards in the frequency range $2-150 \mathrm{kHz}$ and has been identified as a major problem for already some time (Jäkel, 2004; Strzelecki and Benysek, 2008; Smolenski, 2012). As a remedy, a new basic standard IEC 61000-4-19 is currently under discussion which describes test procedures for immunity to conducted, differential mode disturbances in the frequency range $2-150 \mathrm{kHz}$ (IEC 61000-4-19, 2014). The test procedures are defined in frequency domain and utilize predefined frequency steps in order to pass from one test frequency to another. This follows the usual pattern of EMC testing in frequency domain.

In this paper we contribute to the current discussion and point out that testing in time domain is a meaningful addition to the frequency domain test methods that already are suggested. It is noted that the parameters of frequency domain testing are chosen according to physical resonance models. Smart grid components, however, are mainly communication devices that, due to their digital nature, do not necessarily follow resonance patterns. They may exhibit narrowband susceptibilities that can be missed if, during frequency testing, the frequency steps are chosen as too large. In contrast, time domain pulses exhibit frequency spectra which continuously cover frequency intervals, such that narrowband susceptibilities are less likely to be missed.

In the following we will focus on smart meter devices and review in Sect. 2 corresponding EMC standards that at present are in effect. In Sect. 3 the relation of frequency domain testing to physical models is pointed out. By the example of an actual smart meter test it is shown that the susceptibility of a smart meter can be very narrowband. As alternative test pulses in time domain, the well-known damped sinusoidal pulses are introduced in Sect. 4. In contrast to civil standardization, military standards, such as MIL-STD 461, already suggest to apply these pulses in the low frequency 
regime $2-150 \mathrm{kHz}$. As a conclusion, it is recommended to follow MIL-STD 461 and to apply damped sinusoidal pulse testing also in the low-frequency regime to smart meters.

\section{EMC standards for smart meter immunity testing}

Smart meters, as an important class of smart grid component, have to comply to the product standard IEC 62052-11. This standard refers to the following EMC basic standards (IEC 62052-11, 2003):

- Standard IEC 61000-4-2, related to immunity testing with respect to electrostatic discharge (IEC 61000-4-2, 2008).

- Standard IEC 61000-4-3, related to immunity testing with respect to external radiated electromagnetic fields in the frequency range $80 \mathrm{MHz}$ to $2 \mathrm{GHz}$ (IEC 61000-43, 2010).

- Standard IEC 61000-4-4, related to immunity testing with respect to fast transients and bursts, as produced by fast switching processes (IEC 61000-4-4, 2012).

- Standard IEC 61000-4-5, related to immunity testing with respect to transients of a double exponential form, as produced by lightning or slow switching processes (IEC 61000-4-5, 2005).

- Standard IEC 61000-4-6, related to immunity testing with respect to conducted disturbances in the frequency range $150 \mathrm{kHz}$ to $80 \mathrm{MHz}$ (IEC 61000-4-6, 2008).

- Standard IEC 61000-4-12, related to immunity testing with respect to non-repetitive damped sinusoidal transients that also are called "ring waves". The test frequencies of these sinusoidal transients are given by $100 \mathrm{kHz}$ and $1 \mathrm{MHz}$ (IEC 61000-4-12, 2006).

Concerning immunity testing with respect to conducted disturbances, it is noted that the relevant basic standard IEC 61000-4-6 only covers the frequency range $150 \mathrm{kHz}$ to $80 \mathrm{MHz}$. A closer inspection of this standard additionally reveals that only common mode signals are applied to the test objects, while in typical energy distribution networks it is the differential mode which also is relevant (Strzelecki and Benysek, 2008; Paul, 2006).

To also take into account conducted differential mode disturbances in the frequency range below $150 \mathrm{kHz}$, the draft standard IEC 61000-4-19 "Testing and measurement techniques - Test for immunity to conducted, differential mode disturbances in the frequency range from $2 \mathrm{kHz}$ to $150 \mathrm{kHz}$, at a.c. ports" is currently under discussion. To the time of writing it is not known when this standard will be finalized and whether it will be made obligatory for smart meter devices, such that at present there is no standardized test procedure in effect which guarantees immunity of smart meter de- vices with respect to conducted disturbances in the frequency range $2-150 \mathrm{kHz}$.

\section{Immunity tests in frequency domain and the quality factor of a complex system}

From the viewpoint of EMC modelling, a smart meter device is not a canonical structure, such as a simple transmission line or antenna, but rather a complex system. If linearity and time-invariance can be assumed, the immunity of a complex system is characterized by a transfer function $H(s)$ which relates an output quantity, such as an observable $O(s)$ of an operational state, to an input quantity, such as an applied current $I(s)$. In the resonance region, transfer functions are properly discussed in the framework of the singularity expansion method (SEM) and written in the general form (Baum, 1976)

$H(s)=\frac{O(s)}{I(s)} \approx \sum_{m=1}^{M}\left(\frac{R_{m}}{s-s_{m}}+\frac{R_{m}^{*}}{s-s_{m}}\right)$

where the approximation sign indicates that in general the relation is not a mathematically exact equality but of sufficient accuracy if $M$ dominant poles $s_{m}$ of the system are taken into account. These poles represent the frequencies where the system considered is susceptible to the input quantity $I(s)$. The variable $s$ denotes the Laplace variable in the complex plane and the variable $R_{m}$ denotes the residue associated to the $m^{\text {th }}$ resonance pole

$s_{m}=\sigma_{m}+j \omega_{m}$.

The real part $\sigma_{m}$ of the pole, for passive systems, is negative and represents losses of the system, the imaginary part $\omega_{m}$ is the resonance frequency of the pole. If the representation (1) is compared to the transfer function of a damped harmonic oscillator, such as an $R L C$ resonant circuit, it is seen after some analysis that the variables $\sigma_{m}$ and $\omega_{m}$ can also be expressed in terms of an Eigenfrequency $\omega_{0, m}$ and a quality factor $Q_{m}$. The corresponding relations are given by (Chauveau et al., 2007)

$\omega_{0, m}=\left|s_{m}\right|, \quad Q_{m}=-\frac{\omega_{0, m}}{2 \sigma_{m}}$

These notions and concepts are important in order to correctly interpret immunity tests in frequency domain (Gronwald et al., 2012).

As an example, in Fig. 1 the frequency dependency of the received power of a small dipole antenna is displayed in the vicinity of resonance, serving as an elementary model of an EMC victim. At resonance, the received power attains a maximum and the susceptibility of this system becomes, in this frequency range, largest as well. For reliable immunity testing it is decisive to hit the maxima of susceptibility and this, in turn, requires choosing the frequency steps accordingly. If 


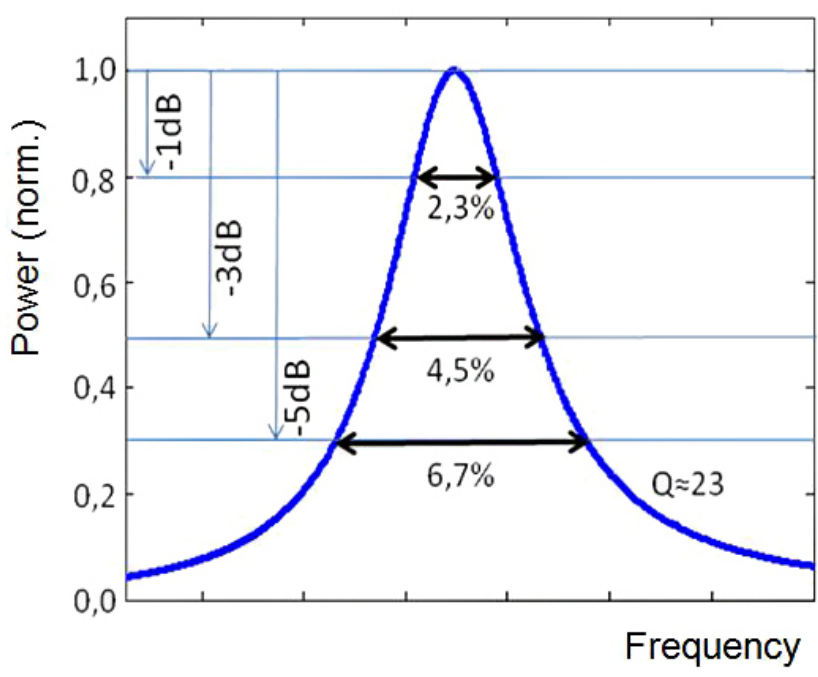

Figure 1. The diagram shows the received power of a matched, small dipole antenna of quality factor $Q=23$. It can be noted that the quality factor is given by the reciprocal of the percentage of the $3 \mathrm{db}$-bandwidth relative to the resonance frequency, i.e., $Q=23 \approx 1 /(0.045)$

testing with a margin of $1 \mathrm{~dB}$ with respect to maximum susceptibility is desired this requires, in the example of Fig. 1, to choose frequency steps according to a $2.3 \%$ rule, that is

$f_{n+1}=f_{n} \cdot 1,023$

as can be seen from Fig. 1 where the $2.3 \%$ bandwidth is indicated. This measure is connected to the quality factor $Q=23$ of the system, the reciprocal of which is equal to the $3 \mathrm{~dB}$ bandwidth. This is also indicated in Fig. 1.

In the draft standard IEC 61000-4-19 frequency steps are prescribed according to a $2 \%$ rule. This allows reliable testing of systems up to a quality factor of $Q=26$ if a $1 \mathrm{~dB}$ margin is desired. For higher quality factors it is then possible to miss frequencies where susceptibility is at a maximum and, as a consequence, immunity at a minimum.

For smart meter devices it is not immediate to determine a quality factor on the basis of physical models. Clearly, smart metering involves digital signal processing and this is not directly accessible to electromagnetic theory. Within a smart metering device analog signals are readily converted to digital signals and then further processed. Disturbances in the frequency range $2-150 \mathrm{kHz}$ may affect this process due to insufficient low-pass filtering and aliasing effects but there is no corresponding general rule which applies to any smart meter.

As an example, an actual smart meter which passed the current EMC standards but turned out to be susceptible during operation has been investigated by means of an immunity test setup as sketched in Fig. 2. A rather small frequency step width of about $0.4 \%$, which is well below the standard fre-

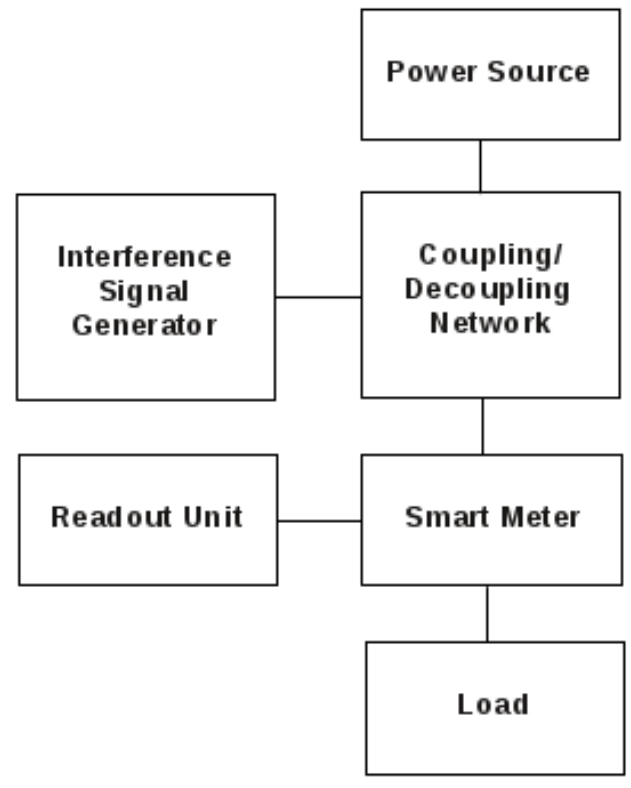

Figure 2. General setup of a smart meter immunity test. It follows the usual pattern of conducted immunity testing (Paul, 2006). The draft standard IEC 61000-4-19 gives more details on how to specify the necessary components that are shown in the figure.

quency step width $2 \%$, was chosen during the test, yielding the result curve of Fig. 3.

The test result exhibits narrowband susceptibilities that exceed acceptable accuracy limits by order of magnitudes. In this case, the frequency step width of $0.4 \%$ turned out to be necessary to recognize the corresponding peaks. It follows from this example that the detection of susceptibilities of actual smart meter devices can require frequency steppings that are below $2 \%$, i.e., below the step width suggested in the draft standard IEC 61000-4-19.

\section{Time domain test procedures and damped sinusoidal pulses}

Time domain test procedures by means of damped sinusoidal pulses have been accomodated in a number of EMC standards, such as in the general MIL-STD-461 (MIL-STD461F, 2007). In this standard the damped sinusoidal pulse mathematically is described in the test procedure CS116 by

$i(t)=I_{0} e^{-\pi f_{0} t / D} \sin \left(2 \pi f_{0} t\right)$

where $D$ indicates a damping factor which has to assume a value of $15 \pm 5$. For a frequency $f_{0}=10 \mathrm{kHz}$, a damping factor of $D=15$, and initial current amplitude $I_{0}=1 \mathrm{~A}$ the resulting current function $i(t)$ is plotted in Fig. 4. The corresponding current spectrum is expressed by the Fourier 


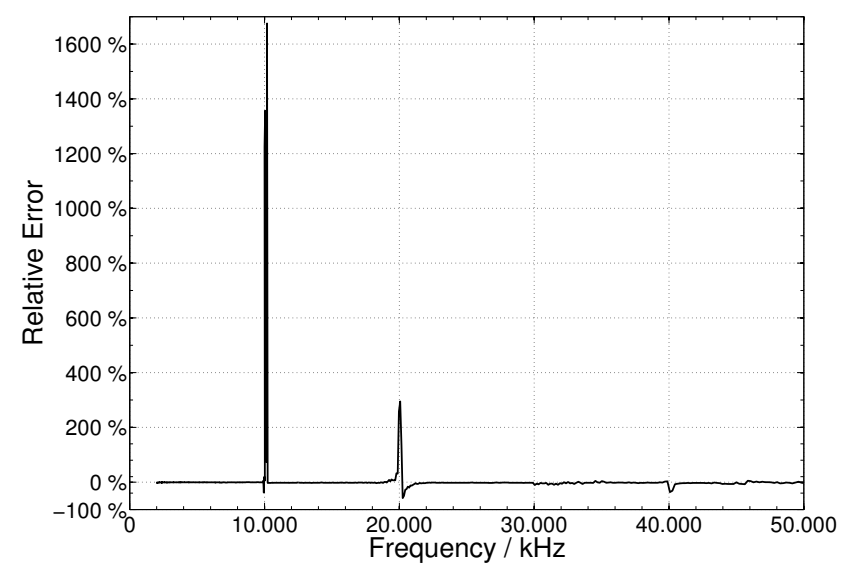

Figure 3. Result of immunity testing of a smart meter device that passed the current EMC tests but nevertheless failed during operation in an actual energy network. Two pronounced peaks at $10 \mathrm{kHz}$ and $20 \mathrm{kHz}$ show unacceptable susceptibilities in the frequency range $2-150 \mathrm{kHz}$, where sinusoidal differential mode test signals were applied.

transform

$I(f)=\frac{1}{2 \pi f_{0}} \frac{I_{o}}{1+\left(\frac{1}{2 D}+j \frac{f}{f_{0}}\right)^{2}}$.

This spectrum can be put on a logarithmic scale according to

$I(f)\left(\mathrm{dB}_{\mathrm{As}}\right)=20 \log (|I(f)| / \mathrm{As})$

and is shown in Fig. 4 as well.

It is seen from Eq. (6) and Fig. 4 that the spectrum peaks at the frequency $f_{0}$ and smoothly decays towards lower and higher frequencies. Clearly, there are no frequency gaps in the spectrum such that is is less likely that narrowband susceptibilities are missed.

In the standard MIL-STD-461 compliance of equipment under test shall be demonstrated in the frequency range $10 \mathrm{kHz}$ to $100 \mathrm{MHz}$ at selected frequencies (MIL-STD-461F, 2007). The civil standard (IEC 61000-4-12, 2006) that applies to smart meters only requires damped sinusoidal pulses at test frequencies $100 \mathrm{kHz}$ and $1 \mathrm{MHz}$. For these pulses the corresponding amplitude spectra decay towards lower frequencies, compare Eq. (6), such that the frequency interval $2-150 \mathrm{kHz}$ is not reasonably covered towards its lower end. In view of both the presence of considerable disturbances in the complete frequency interval $2-150 \mathrm{kHz}$ that are known to effect smart meters and possible narrowband susceptibilities it is therefore suggested to also apply the immunity criteria of MIL-STD-461 to smart meter devices and to extend time domain testing by means of damped sinusoidal pulses towards lower frequencies down to at least $10 \mathrm{kHz}$.
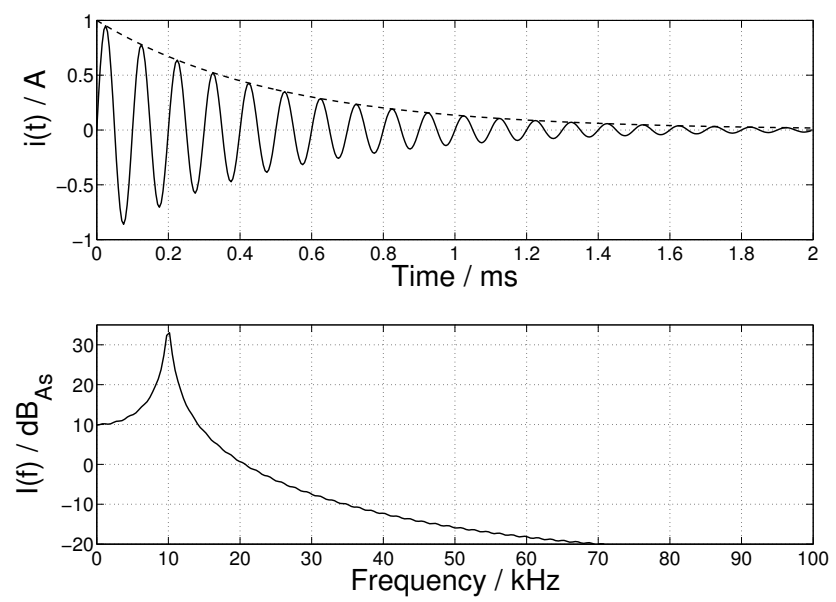

Figure 4. Damped sinusoidal pulse of frequency $f_{0}=10 \mathrm{kHz}$ and its associated spectrum.

\section{Conclusions}

At present, EMC standards for smart meter and other smart grid devices still are under discussion to better take into account the increasing occurrences of conducted disturbances in the frequency range $2-150 \mathrm{kHz}$. The discussions focus on frequency domain test methods which are aimed to test within the resonance region of a system. However, smart grid devices mainly are digital systems such that physical resonance models do not necessarily apply. It has been shown by the example of an immunity test of a particular smart meter that corresponding susceptibilies can be very narrowband and can be missed during a frequency sweep if the step width is too large. Therefore it is recommended to complement immunity testing of smart grid devices by time domain tests and associated continuous amplitude spectra along the lines of MIL-STD-461.

Acknowledgements. The author thanks EWE AG Oldenburg for providing smart meter devices for investigation. Measurement support of EMC Services TUEV Nord by T. Weber also is gratefully acknowledged.

Edited by: F. Sabath

Reviewed by: T. Ehlen, H. Garbe, and F. Sabath

\section{References}

Baum, C. E.: The Singularity Expansion Method, in: Transient Electromagnetic Fields, edited by: Felsen, L. B., Springer, Heidelberg, 129-179, 1976.

CENELEC SC 205A: Study Report on Electromagnetic Interference between Electrical Equipment/Systems in the Frequency Range below 150 kHz, SC205A/SEC0260/R, April, 2010. 
CENELEC SC 205A: Study Report on Electromagnetic Interference between Electrical Equipment/Systems in the Frequency Range below 150 kHz, Ed. 2, SC205A/SEC0339/R, April, 2013.

Chauveau, J., de Beaucoudrey, N., and Saillard, J.: Characterization of Perfectly Conducting Targets in Resonance Domain with their Quality of Resonance, Progress in Electromagnetic Research, 46, 165-188, 2007.

Gronwald, F., Stadler, T., and Kebel, R.: Compensation of Unavailable Test Frequencies during Immunity Measurements, in: Proceedings of ESA Workshop on Aerospace EMC, (ESA, SP-702), 2012.

IEC 61000-4-2: Electromagnetic compatibility (EMC) - Part 4-2: Testing and measurement techniques - Electrostatic discharge immunity test, 2008.

IEC 61000-4-3: Electromagnetic compatibility (EMC) - Part 43: Testing and measurement techniques - Radiated, radiofrequency, electromagnetic field immunity test, 2010.

IEC 61000-4-4: Electromagnetic compatibility (EMC) - Part 44: Testing and measurement techniques - Electrical fast transient/burst immunity test, 2012.

IEC 61000-4-5: Electromagnetic compatibility (EMC) - Part 45: Testing and measurement techniques - Surge immunity test, 2005.

IEC 61000-4-6: Electromagnetic compatibility (EMC) - Part 4-6: Testing and measurement techniques - Immunity to conducted disturbances, induced by radio-frequency fields, 2008.
IEC 61000-4-12: Electromagnetic compatibility (EMC) - Part 4-12: Testing and measurement techniques - Ring wave immunity test, 2006.

IEC 61000-4-19: Electromagnetic compatibility (EMC) - Part 419: Testing and measurement techniques - Test for immunity to conducted, differential mode disturbances in the frequency range from $2 \mathrm{kHz}$ to $150 \mathrm{kHz}$ at a.c. ports, in preparation, 2014.

IEC 62052-11: Electricity metering equipment (AC) - General requirements, tests and test conditions - Part 11: Metering equipment, 2003.

Jäkel, B.: Störungen auf industriellen Niederspannungsnetzen im Frequenzbereich bis $150 \mathrm{kHz}$, in: Elektromagnetische Verträglichkeit EMV 2004, VDE Verlag, Berlin, 743-750, 2004.

Kirchhof, J.: Grenzwertlücke - Wechselrichter stört Elektrizitätszähler, in: Elektromagnetische Verträglichkeit EMV 2010, VDE Verlag, Berlin, 97-104, 2010.

MIL-STD-461F: Requirements for the control of electromagnetic interference characteristics of subsystems and equipment, 2007.

Paul, C. R.: Introduction to Electromagnetic Compatibility, 2nd ed., Wiley, New Jersey, USA, 2006.

Smolenski, R.: Conducted Electromagnetic Interference (EMI) in Smart Grids, Springer, London, 2012.

Strzelecki, R. and Benysek, G. (Eds.): Power Electronics in Smart Electrical Energy Networks, Springer, London, United Kingdom, 2008. 